\title{
Direkter Draht zwischen Praxis und Labor kostenlos
}

Die VITA Easyshade-Technologie für die digitale Zahnfarbbestimmung hat sich seit über 10 Jahren in Zahnarztpraxis und Labor etabliert. Um jetzt auch Anwendern von mobilen AppleGeräten die komfortable digitale Farbkommunikation mit dem

VITA Easyshade V aus der Praxis in das Labor zu ermöglichen, steht ab sofort die App VITA mobileAssist für iOS kostenlos zum Download im Apple App Store bereit. Nach der digitalen Zahnfarbbestimmung mit dem VITA Easyshade V am Patienten kann die Farbinformation mit einem Knopfdruck bequem per Bluetooth jetzt auch an mobile Apple-Geräte übertragen werden. Dort werden die Ergebnisse der Zahnfarbmessung automatisch am Patientenbild angezeigt, die entweder direkt über die App aufgenommen oder von extern importiert werden können. Das Messergebnis kann mithilfe der App im Standardfarbsystem VITA classical A1-D4, VITA SYSTEM 3D-MASTER sowie für VITABLOCS- und Bleachedfarben auf dem Display dargestellt werden. Ausführliche Informationen unter www.vita-zahnfabrik.com.

Nach einer Pressmitteilung der

VITA Zahnfabrik H. Rauter GmbH \& Co. KG,

Bad Säckingen 